\title{
Enhanced Model Reference Adaptive Control based on Data-driven Technique for Permanent Magnet Synchronous Motors with Uncertainties
}

\author{
Feng Qiao ${ }^{1, *}$, Zhizhen Liu ${ }^{1}$ and Kun Zong ${ }^{2}$ \\ ${ }^{1}$ School of Electrical Engineering, Shandong University, Jinan 250061, China \\ ${ }^{2}$ UFR Sciences et Techniques, Université du Havre, Le Havre 76600, France
}

Received 22 March 2017; Accepted 5 August 2017

\begin{abstract}
Precise velocity control of permanent magnet synchronous motors (PMSMs) is challenging due to the uncertainties induced by the mathematical model, unmodeled dynamics, and various disturbances; consequently, the traditional model reference adaptive control (MRAC) for PMSMs becomes questionable. In order to improve the traditional MRAC and lessen the influence of various sources of uncertainty, this study proposes an enhanced MRAC approach based on datadriven technique. First, the approach established an equivalent differential expression linear model for the discrete-time nonlinear description of PMSM and designed a data-driven controller using a model-free adaptive technique. This design depended on the unique bounded parameter referred as pseudo-partial-derivative (PPD) that was a slowly time-varying parameter relating to the system action point or system dynamics. Second, the approach identified the parameter PPD using Popov criterion to ensure the asymptotic stability of the controlled system. Finally, the approach was simulated and applied on a $50 \mathrm{~kW}$ PMSM drive of Toyota Prius II hybrid electric vehicles to demonstrate the effects of different control parameters. Results show that the proposed approach does not suffer from the drawback of the modeling process and unmodeled dynamics and provides improved velocity-tracking precision against parameter variations and external disturbances. When the weight factor increases from 0.1 to 1 or the pseudo-orders from 1 to 5 , the velocity presents high robustness. Moreover, the approach yields satisfactory disturbance rejection and fault tolerance compared with the traditional MRAC even if some input and output (I/O) data are missing. This study solves the problem of dependence on plant models for the traditional MRAC and achieves better suppression of uncertainties, demonstrating the effective applications of this approach for actual nonlinear motor systems with uncertainties.
\end{abstract}

Keywords: PMSM, MRAC, Data-driven, Model-free, Popov

\section{Introduction}

Permanent magnet synchronous motors (PMSMs) are widely used in high-performance servo applications owing to their high efficiency, superior power density, and large torque-toinertia ratio. Nowadays, PMSM driving has become the trend in electric drive control systems with the support of rich rare earth materials and advanced control strategies [1]. However, PMSMs are nonlinear multivariable dynamic systems, and the uncertainties induced by parameter variations, external disturbances, and unmodeled dynamics have become a common problem, which reduces the stability and robustness of the controlled system. To suppress these uncertainties and achieve the desired servo control performance, advanced strategies must be developed.

Traditional proportional-integral-derivative (PID) control can achieve better performance when parameters effectively match. However, PID shows poor performance when parameters vary or when affected by external disturbances; moreover, it does not consider dynamic response and disturbance rejection ability coordinately.

Adaptive control is a popular approach to solve these

\footnotetext{
*E-mail address: qfeng0810@163.com

ISSN: 1791-2377 @ 2017 Eastern Macedonia and Thrace Institute of Technology. All rights reserved. doi:10.25103/jestr.104.10
}

problems, and one of the most typical representatives is Model Reference Adaptive Control (MRAC) [2]. However, this approach typically assumes that the mathematical model of the system is known and the parameters are unknown or slowly time-varying [3]. For practical PMSM drives, the models are often complex and the parameters cannot be easily identified; thus, this adaptive control is questionable.

To solve these problems, the present study focuses on the investigation of enhanced MRAC and attempts to achieve a general approach to control dynamical PMSM systems with uncertainties.

\section{State of the art}

Despite the advantages of PMSM drives, high precision velocity control is challenging because the mathematical model of PMSM only approximates the actual plant, and the motion dynamics of PMSM are complicated, intrinsically nonlinear, and subject to various sources of disturbance and uncertainty. Classical or modern control theories cannot satisfy the high performance requirements in practical applications due to the preconditions of precisely or partially known mathematical models. For such problems, adaptive control is an effective solution [4]. Adaptive control allows the systems to operate automatically at optimal or 
approximately optimal conditions even if the model is incomplete or partially known. Among numerous adaptive control approaches, the MRAC should be first considered because of its improved theoretical research results and practical applications. For PMSM systems with uncertainties, the MRAC presents strong robustness and favorable disturbance rejection properties against structure or parameter variations. In the past decades, the research and design of MRAC systems have significantly progressed [4], [5]. Relatively complete and comprehensive guidelines are now available for designing and implementing adaptive controllers.

However, traditional MRAC has been having drawbacks from mathematical models, external disturbances, and unmodeled dynamics, especially for controlling nonlinear time-varying PMSM systems or random processes. Researchers have recognized that the crucial task of enhanced MRAC for PMSM systems is to reject various external disturbances and improve robustness in various uncertainties. Landau et al. proposed a direct feedback adaptive regulation scheme to suppress multiple unknown and/or time-varying vibrations, and minimized the residual force by applying an appropriate control effort through the internal model principle and Youla-Kucera parameterization [6]. However, performance is affected by the model and unmodeled dynamics because this approach introduced a dynamic model for external disturbances to the controller. In fact, the effects of disturbances or uncertainties are related to the variations of the generalized error of MRAC. In an ideal case, when no disturbance or unmodeled dynamics is present, the tracking error converges to zero. In a non-ideal case, the mean-square tracking error is of the order of magnitude of the modeling error provided by the unmodeled dynamics that satisfies a norm-bound condition [7]. Therefore, a prediction-identification model based adaptive control method was proposed for uncertain systems with timevarying parameters in the presence of bounded external disturbances, and the desired tracking performance was achieved by feeding back the state prediction error to the identification model. The desired closed-loop properties were obtained when the error feedback gain was proportional to the square root of the adaptation rate [8]. However, an apparent drift of adaptive gains may occasionally arise, eventually leading to closed-loop instability. For the drift of adaptive gains, a parameter projection algorithm can be used to solve the problem [9]. $\mathrm{Gao}$ et al. investigated the robust indirect MRAC with the normalized adaptive law for a class of discrete-time systems with unmodeled dynamics and bounded disturbances [10], but the constructed parameter estimation algorithm was affected by the modeling process.

Improved performances were achieved for the PMSM control system; however, single nonlinear approach was often applicable to solve a certain problem. Therefore, the researchers were committed to seek valid integrated nonlinear control techniques, and numerous new branches of MRAC have emerged. At present, the neural network-based MRAC approach [11], [12], [13] and fuzzy MRAC approach [14], [15] are two successful integrated approaches. The former adopted the neural network learning algorithm as the adaptive control algorithm and trained the adjustment parameter on-line or off-line with the neural network technique. Compared with the traditional MRAC, the approach leads to improved rejection of external disturbances and enhances the robustness. However, the online or off-line train process is complex and implies the structure information of the controlled plant. The fuzzy MRAC approaches used the fuzzy basis function expansion to represent the unknown parameters and changed the identification problem from identifying the original unknown parameters to identifying the coefficients of fuzzy basis function expansion. The approach provides higher adaptation ability than the basic adaptive control and solves the problem of stability of nonlinear systems with parameter uncertainties. However, selecting of fuzzy rules and membership functions depends on a priori knowledge. Moreover, variable structure model reference adaptive controls [16] can improve the robustness and fault tolerance and ensure disturbance rejection properties, but the chatter has always been the main obstacle of the approach application. These integrated strategies have a common problem that is subject to models with known structure, rules, training process, or prior knowledge. When the controlled plant is unknown or difficult to describe with mathematical forms, the strategies cannot be used. Furthermore, if the approaches for a specific plant are extended to a new actual system, the performance becomes unpredictable.

Actually, the input and output (I/O) information are significant for studying the behavior and uncertainties of PMSM. Thus, we were motivated to study data-driven control approaches, which mainly concentrate on the importance of $\mathrm{I} / \mathrm{O}$ information and design controller that merely use I/O data of a plant. These approaches do not require explicit models or structural information of the plant; thus, the modeling process and unmodeled dynamics disappear, whereas the parameter variations and external disturbances are suppressed. Several data-driven control approaches, such as simultaneous perturbation stochastic approximation control, multi-level recursive control, modelfree adaptive control, unfalsified control, iterative feedback tuning, virtual reference feedback tuning, and lazy learning, are currently available [17]. Compared with other approaches, the model-free adaptive control offers low computational burden, easy implementation, and strong robustness; thus, it is suitable for many practical applications [18]. The model-free adaptive control can design a common controller for a class of industrial process based on the realtime I/O information and does not require any external measurement or training process. Wang et al. proposed a second-order universal model-free adaptive controller, the parameters of which were optimized by a gradient descent algorithm [19]. Jin and Qiao designed a higher-order modelfree adaptive controller for a class of nonlinear systems that could obtain promising results using only I/O information [20], [21]. However, these designs do not fully utilize the historical $\mathrm{I} / \mathrm{O}$ information of the controlled plant and use incomplete or partial missing information, thereby causing poor robustness, oscillation, and instability of these systems. Model-free predictive control [22], [23] and model-free iterative learning control [24], [25], have been developed and integrated model-free adaptive control with advanced control strategies to improve their performance. However, the model-free predictive control greatly depends on the prediction accuracy of the model, and the model-free iterative learning control is limited to the plant of trial repetition in finite time intervals.

The main work of this study attempts to improve the traditional MRAC with the model-free adaptive technique and proposes a data-driven MRAC approach for PMSM with uncertainties. An equivalent differential expression linear model was built with a time-varying parameter called pseudo-partial-derivative (PPD) in the sense of $\mathrm{I} / \mathrm{O}$ 
equivalence. This parameter was identified by the output error between the reference model and practical model based on the Popov criterion. Simulations were provided to illustrate the effectiveness of the proposed approach when applied to a PMSM system.

The remainder of this study is organized as follows. Section 3 describes the data-driven MRAC approach that designs an adaptive control law based on the equivalent linear model for a discrete-time nonlinear PMSM system and presents the parameter estimation algorithm based on the Popov criterion. Section 4 presents a simulation on a 50 kW PMSM drive of Toyota Prius II hybrid electric vehicles to illustrate the effectiveness and superior performance of the data-driven MRAC approach. Section 5 summarizes the conclusions.

\section{Methodology}

3.1 Problem formulation and nonparametric dynamic linearization method

The control system of PMSM can be described by the following discrete-time single-input single-output (SISO) nonlinear form.

$$
\omega(k+1)=f\left(\omega(k), \cdots, \omega\left(k-L_{y}\right), T_{e}(k), \cdots, T_{e}\left(k-L_{u}\right)\right)
$$

where $\omega(k) \in R^{m}$ and $T_{e}(k) \in R^{m}$ are the output and input at time $k$, respectively; $L_{u}$ and $L_{y}$ are the unknown orders (pseudo-orders), and $f(\cdots)$ is an unknown nonlinear function.

To guide our discussion, we developed the following assumptions:

Assumption 1: Input and output of system (1) are observable and controllable, that is, for the desired bounded output signal $\omega^{*}(k+1)$, there exists a bounded feasible input signal that makes the practical output equal to the desired output.

Assumption 2: Partial derivatives of $f(\cdots)$ with respect to control input $T_{e}(k)$ are existent and continuous.

Assumption 3: System (1) presents the generalized Lipschitz condition, that is, $|\Delta \omega(k+1)| \leq c\left|\Delta T_{e}(k)\right|$ for any $k$, where $\Delta \omega(k+1)=\omega(k+1)-\omega(k), \Delta T_{e}(k)=T_{e}(k)-T_{e}(k-1)$, $\left|\Delta T_{e}(k)\right| \neq 0$, and $c$ is a positive constant.

For the nonlinear system (1) that satisfies assumptions 1, 2, and 3, a PPD vector $\theta(k)$ and $|\theta(k)| \leq b$ must exist, when $\left|\Delta T_{e}(k)\right| \neq 0$ for all $k$, such that system (1) can be transformed into the following equivalent linear model.

$\Delta \omega(k+1)=\theta(k) \Delta T_{e}(k)$

Equation (2) is defined as the universal linear model of system (1) based on I/O information increment expression, which converts a complex SISO nonlinear system into a linear system with time-varying parameter, $\theta(k)$.
3.2 Design of control law based on data-driven technique The objective of the proposed control approach is to find a suitable control input sequence $T_{e}(k)$ to achieve no difference tracking with the given desired trajectory, $\omega^{*}(k)$.

Considering the following control criterion function:

$$
\begin{aligned}
J\left(T_{e}(k), a_{i}, b_{j}\right)= & \left|\sum_{i=1}^{L_{y}} a_{i}\left(\omega^{*}(k-i+2)-\omega(k-i+2)\right)\right|^{2} \\
& +\lambda\left|\sum_{j=1}^{L_{u}} b_{j}\left(T_{e}(k-j+1)-T_{e}(k-j)\right)\right|^{2}
\end{aligned}
$$

where $\lambda$ is a positive weight factor that restricts the variation of control inputs. The first and the second items in equation (3) denote the weighted output error and weighted input error, respectively, of previous $i$ or $j$ sampling instants, which are known at the $k$ sampling instant. $a_{i}$ and $b_{j}$ are the weight factors, which directly determine the used region and used degree of previous $\mathrm{I} / \mathrm{O}$ information, respectively. $\quad a_{i}=\left(a_{1}, a_{2}, \mathrm{~L}, a_{L_{y}}\right)^{T} \quad$ with $\quad \sum_{i=1}^{L_{y}} a_{i}=1$, $b_{j}=\left(b_{1}, b_{2}, \mathrm{~L}, b_{L_{u}}\right)^{T}$ with $\sum_{j=1}^{L_{u}} b_{j}=1$.

Using the optimal condition $\frac{1}{2} \frac{\partial J\left(T_{e}(k), a_{i}, b_{j}\right)}{\partial T_{e}(k)}=0$, yields the following:

$$
\begin{aligned}
T_{e}(k) & =T_{e}(k-1)+\frac{a_{1} \theta(k)}{\lambda b_{1}^{2}+a_{1}^{2} \theta^{2}(k)}\left(a_{1}\left(\omega^{*}(k+1)-\omega(k)\right)\right. \\
& \left.+\sum_{i=2}^{L_{y}} a_{i}\left(\omega^{*}(k-i+2)-\omega(k-i+2)\right)\right) \\
& -\frac{\lambda b_{1}}{\lambda b_{1}^{2}+a_{1}^{2} \theta^{2}(k)} \sum_{j=2}^{L_{u}} b_{j} \Delta T_{e}(k-j+1)
\end{aligned}
$$

Remark 1: The control criterion function (3) differs from the general function in [17]. This function contains not only the control inputs included in a $L_{u}$ (input pseudoorders) sliding time window before the current instant but the output error in a $L_{y}$ (output pseudo-orders) sliding time window before the current sampling instant. This function can satisfy the different control requirements of complex systems by selecting suitable values of $L_{u}$ and $L_{y}$ to achieve high freedom and accuracy.

Remark 2: The control law (4) was designed merely using the $\mathrm{I} / \mathrm{O}$ measurement data of the controlled plant; it has a recursive form. It is different from the control law in [17] and has no relations with any explicit model dynamics and structural information of the plant.

\subsection{Parameter estimate based on Popov criterion}

Complex behaviors of the controlled plant, such as nonlinear, parameter variations and unmodeled dynamics, are integrated into the time-varying parameter $\theta(k)$. Therefore, the dynamic characteristics of $\theta(k)$ may be extremely complex and cannot be easily mathematically described, but their values may not be sensitive to time-varying factors and can easily be estimated. 
An adaptive mechanism was employed in this study to estimate $\theta(k)$ and cause the generalized error to converge to zero, considering that MRAC can adjust the controller parameters according to the error between the reference model and practical model.

The second order Auto-Regressive integrated Moving Average (ARMA) model of PMSM is described as follows, when the load is treated as disturbance:

$$
\omega(k+1)=(1+a) \omega(k)-a \omega(k-1)+b \Delta T_{e}(k)
$$

where $a=e^{-B T / J}, b=\left(1-e^{-B T / J}\right) / B ; B$ is the viscous friction coefficient, $J$ is the rotational inertia, and $T$ is the sample period.

The reference model is set as follows:

$$
\omega_{m}(k+1)=a_{m} \omega_{m}(k)+b_{m} \omega_{m}(k-1)+c_{m} r(k)+d_{m} r(k-1)
$$

where $\omega_{m}(k)$ is the output of the reference model and $r(k)=\omega^{*}(k)$ is the input of reference model.

To overcome the one-step delay of the discrete algorithm, a priori variable and a posteriori variable are introduced:

$$
\begin{aligned}
\omega^{0}(k+1)= & (1+a) \omega(k)-a \omega(k-1) \\
& +\frac{a_{1} b \theta(k-1)}{\lambda b_{1}^{2}+a_{1}^{2} \theta^{2}(k-1)}\left(a_{1}\left(\omega^{*}(k+1)-\omega(k)\right)\right. \\
& \left.+\sum_{i=2}^{L_{y}} a_{i}\left(\omega^{*}(k-i+2)-\omega(k-i+2)\right)\right) \\
& -\frac{\lambda b_{1} b}{\lambda b_{1}^{2}+a_{1}^{2} \theta^{2}(k-1)} \sum_{j=2}^{L_{u}} b_{j} \Delta T_{e}(k-j+1)
\end{aligned}
$$

$$
\begin{aligned}
\omega(k+1)= & (1+a) \omega(k)-a \omega(k-1) \\
& +\frac{a_{1} b \theta(k)}{\lambda b_{1}^{2}+a_{1}^{2} \theta^{2}(k)}\left(a_{1}\left(\omega^{*}(k+1)-\omega(k)\right)\right. \\
& \left.+\sum_{i=2}^{L_{y}} a_{i}\left(\omega^{*}(k-i+2)-\omega(k-i+2)\right)\right) \\
& -\frac{\lambda b_{1} b}{\lambda b_{1}^{2}+a_{1}^{2} \theta^{2}(k)} \sum_{j=2}^{L_{u}} b_{j} \Delta T_{e}(k-j+1)
\end{aligned}
$$

The priori and posteriori generalized errors are as follows:

$$
\begin{aligned}
& e^{0}(k+1)=\omega_{m}(k+1)-\omega^{0}(k+1) \\
& e(k+1)=\omega_{m}(k+1)-\omega(k+1)
\end{aligned}
$$

Equation (10) can be rewritten by subtracting (8) from (6), as follows:

$$
e(k+1)=a_{m} e(k)+b_{m} e(k-1)+y(k)
$$

where:

$$
\begin{aligned}
y(k)= & -y_{1}(k)=\left(a_{m}-a-1\right) \omega(k)+\left(b_{m}+a\right) \omega(k-1) \\
& +c_{m} \omega^{*}(k)+d_{m} \omega^{*}(k-1) \\
& -\frac{a_{1} b \theta(k)}{\lambda b_{1}^{2}+a_{1}^{2} \theta^{2}(k)}\left(a_{1}\left(\omega^{*}(k+1)-\omega(k)\right)\right. \\
& \left.+\sum_{i=2}^{L_{y}} a_{i}\left(\omega^{*}(k-i+2)-\omega(k-i+2)\right)\right) \\
& +\frac{\lambda b_{1} b}{\lambda b_{1}^{2}+a_{1}^{2} \theta^{2}(k)} \sum_{j=2}^{L_{u}} b_{j} \Delta T_{e}(k-j+1)
\end{aligned}
$$

A linear compensator is selected as follows:

$$
\begin{aligned}
& v^{0}(k+1)=e^{0}(k+1)+\sum_{i=1}^{2} d_{i} e(k-i+1) \\
& v(k+1)=e(k+1)+\sum_{i=1}^{2} d_{i} e(k-i+1)
\end{aligned}
$$

An equivalent nonlinear time-varying feedback system can be formed using Equations (11) and (14).

According to the Popov criterion, the asymptotic stability of the system must satisfy the two following conditions:

(1) Transfer function of forward channel is strictly positive;

(2) Feedback channel satisfies the Popov integral inequality.

The transfer function of the forward channel is as follows:

$$
G(z)=\frac{v(\mathrm{z})}{y(\mathrm{z})}=\frac{1+d_{1} z^{-1}+d_{2} z^{-2}}{1-a_{m} z^{-1}-b_{m} z^{-2}}
$$

To confirm the strictly positive $G(z)$, the values of $d_{1}$ and $d_{2}$ are selected as follows: $d_{1} \leq \frac{4\left(1+b_{m}\right)}{3+a_{m}+b_{m}}, d_{2}=d_{1}-1$.

Substituting $y(k)$ into Popov inequality, we obtained the following:

$$
\begin{aligned}
\eta\left(0, k_{1}\right)= & \sum_{k=0}^{k_{1}} v(k)\left\{\left(-a_{m}+a+1\right) \omega(k)-\left(b_{m}+a\right) \omega(k-1)\right. \\
& -c_{m} \omega^{*}(k)-d_{m} \omega^{*}(k-1) \\
& +\frac{a_{1} b \theta(k)}{\lambda b_{1}^{2}+a_{1}^{2} \theta^{2}(k)}\left(a_{1}\left(\omega^{*}(k+1)-\omega(k)\right)\right. \\
& \left.+\sum_{i=2}^{L_{y}} a_{i}\left(\omega^{*}(k-i+2)-\omega(k-i+2)\right)\right) \\
& \left.-\frac{\lambda b_{1} b}{\lambda b_{1}^{2}+a_{1}^{2} \theta^{2}(k)} \sum_{j=2}^{L_{u}} b_{j} \Delta T_{e}(k-j+1)\right\} \\
= & \sum_{k=0}^{k_{1}} v(k)\left\{\sum_{i=1}^{L_{y}} L_{i}(\theta(k)) \omega^{*}(k-i+2)\right. \\
& +\sum_{i=1}^{L_{y}} M_{i}(\theta(k)) \omega(k-i+1) \\
& \left.+\sum_{i=1}^{L_{u}} N_{i}(\theta(k)) \Delta T_{e}(k-i)\right\} \\
\geq & -\gamma_{0}^{2}
\end{aligned}
$$


where:

$$
\begin{gathered}
\left\{L_{i}(\theta(k)), i=1,2, \mathrm{~L}, L_{y}\right\}=\left\{\frac{a_{1}^{2} b \theta(k)}{\lambda b_{1}^{2}+a_{1}^{2} \theta^{2}(k)}, \frac{a_{1} a_{2} b \theta(k)}{\lambda b_{1}^{2}+a_{1}^{2} \theta^{2}(k)}-c_{m},\right. \\
\left.\frac{a_{1} a_{3} b \theta(k)}{\lambda b_{1}^{2}+a_{1}^{2} \theta^{2}(k)}-d_{m}, \frac{a_{1} a_{4} b \theta(k)}{\lambda b_{1}^{2}+a_{1}^{2} \theta^{2}(k)}, \mathrm{L}, \frac{a_{1} a_{L_{y}} b \theta(k)}{\lambda b_{1}^{2}+a_{1}^{2} \theta^{2}(k)}\right\} \\
\left\{\begin{aligned}
&\left\{L_{i}(\theta(k)), i=1,2, \mathrm{~L}, L_{y}\right\}=\left\{\frac{a_{1}^{2} b \theta(k)}{\lambda b_{1}^{2}+a_{1}^{2} \theta^{2}(k)}, \frac{a_{1} a_{2} b \theta(k)}{\lambda b_{1}^{2}+a_{1}^{2} \theta^{2}(k)}-c_{m},\right. \\
&\left.\frac{a_{1} a_{3} b \theta(k)}{\lambda b_{1}^{2}+a_{1}^{2} \theta^{2}(k)}-d_{m}, \frac{a_{1} a_{4} b \theta(k)}{\lambda b_{1}^{2}+a_{1}^{2} \theta^{2}(k)}, \mathrm{L}, \frac{a_{1} a_{L_{y}} b \theta(k)}{\lambda b_{1}^{2}+a_{1}^{2} \theta^{2}(k)}\right\} \\
&\left\{M_{i}(\theta(k)), i=1,2, \mathrm{~L}, L_{y}\right\}=\left\{\frac{a_{1}\left(a_{1}+a_{2}\right) b \theta(k)}{\lambda b_{1}^{2}+a_{1}^{2} \theta^{2}(k)}-a_{m}+a+1,\right. \\
& \frac{-a_{1} a_{3} b \theta(k)}{\lambda b_{1}^{2}+a_{1}^{2} \theta^{2}(k)}-b_{m}-a, \frac{a_{1} a_{4} b \theta(k)}{\lambda b_{1}^{2}+a_{1}^{2} \theta^{2}(k)}, \\
&\left.\mathrm{L}, \frac{a_{1} a_{L_{y}} b \theta(k)}{\lambda b_{1}^{2}+a_{1}^{2} \theta^{2}(k)}\right\}
\end{aligned}\right.
\end{gathered}
$$$$
\left\{N_{i}(\theta(k)), i=1,2, \mathrm{~L}, L_{y}\right\}=\left\{\frac{-\lambda b b_{1} b_{2} \theta(k)}{\lambda b_{1}^{2}+a_{1}^{2} \theta^{2}(k)}, \frac{-\lambda b b_{1} b_{3} \theta(k)}{\lambda b_{1}^{2}+a_{1}^{2} \theta^{2}(k)},\right.
$$$$
\left.\mathrm{L}, \frac{-\lambda b b_{1} b_{L_{u}-1} \theta(k)}{\lambda b_{1}^{2}+a_{1}^{2} \theta^{2}(k)}\right\}
$$

To hold Equation (16), the following parameter adaptive estimation law must be selected:

$$
\theta(k)=\theta(k-1)+\frac{\left(\alpha_{1} \omega^{*}(k+1)+\alpha_{2} \omega(k)+\beta \Delta T_{e}(k-1)\right) v(k)}{1+\alpha_{1} \omega^{* 2}(k+1)+\alpha_{2} \omega^{2}(k)+\beta \Delta T_{e}^{2}(k-1)}
$$

where $\alpha_{1}, \alpha_{2}$, and $\beta$ are gain coefficients.

The structure of the data-driven MRAC approach, which is based on this design, is shown in Figure 1. In this approach, a model-free controller is designed with only the I/O information of PMSM to achieve a practical output. The practical output is compared with the output of the reference model, and a measurement of difference between the actual and desired performance is obtained. This information, together with the practical output and the controller output, is used by an adaptive mechanism to directly estimate the real-time parameter $\theta(k)$ to force asymptotically the generalized error to zero. When the output tracking characteristic is influenced by the parameter variations, unmodeled dynamics or external disturbances, the estimator can correct the adjustable parameter $\theta(k)$ real-time to reach the output of the reference model and achieve no difference tracking.

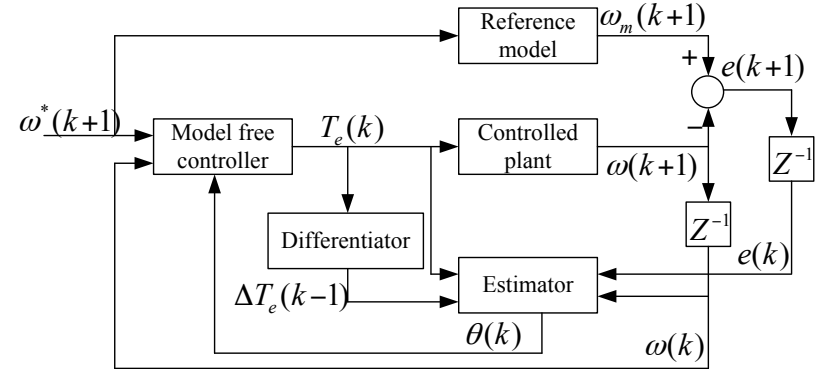

Fig. 1. System structure block diagram based on data-driven MRAC

\section{Result Analysis and Discussion}

In this section, an example on a 50-kW PMSM control problems is presented to show the tracking performance of the proposed data-driven MRAC approach.

\subsection{Data generator of a practical PMSM system}

A practical PMSM served as an I/O data generator to implement the proposed approach. No explicit model and structural information of the PMSM were included in the controller design. [26]:

The nonlinear model of PMSM is described as follows

$$
\left\{\begin{array}{l}
\dot{\theta}_{m}=\omega_{m} \\
m r^{2} \dot{\omega}_{m}=T_{e}-T_{L}-T_{\text {friction }}-T_{\text {ripple }}
\end{array}\right.
$$

where $w_{m}$ and $\theta_{m}$ are the mechanical angular velocity and mechanical angle, respectively. $T_{e}, T_{L}, T_{\text {friction }}$, and $T_{\text {ripple }}$ are the electromagnetic torque, load torque, friction torque, and ripple torque, respectively. $m$ is the slide weight, including load. $r$ is the outer diameter of the rotor.

The friction and ripple torque are assumed to be modeled as follows:

$$
\left\{\begin{array}{l}
T_{\text {friction }}=\left(T_{c}+\left(T_{s}-T_{c}\right) e^{-\left(\omega_{m} / \omega_{m d}\right)^{\delta}}+T_{v} \omega_{m}\right) \operatorname{sgn}\left(\omega_{m}\right) \\
T_{\text {ripple }}=F \sin \left(\omega_{0} \theta_{m}\right)
\end{array}\right.
$$

where $T_{c}$ is the minimum level of coulomb friction torque, $T_{s}$ is the level of static friction torque, $T_{v}$ is the viscous friction torque, $\omega_{m d}$ is the desired angular velocity, $\omega_{0}$ is the angular velocity of the ripple torque, $F$ is the swing of the ripple torque, and $\delta$ is an additional empirical parameter.

Using a practical PMSM system (the parameters of which are listed in Table 1) and discretizing equation (18) yields the following:

$$
\left\{\begin{aligned}
y(k+1)=y(k) & +z(k) \\
\mathrm{z}(k+1)=z(k) & +\frac{1}{1.152}\left(u(k)-8-\left(1.6+1.6 e^{-\left(\mathrm{z}(k) / z_{d}(k)\right)^{2}}\right.\right. \\
& +1.6 z(k)) \operatorname{sgn}(\mathrm{z}(k))-1.6 \sin (900 \mathrm{y}(k)))
\end{aligned}\right.
$$

where $y(k)$ and $z(k)$ are the system outputs denoting $\theta_{m}$ and $w_{m}$, respectively; $u(k)$ is the system control input $T_{e}$. 
$\underline{\text { Table 1. PMSM system parameters }}$

\begin{tabular}{l|c|c|c}
\hline \multicolumn{1}{c|}{ Parameters } & Symbols & Values & Units \\
\hline Rated torque & $T_{N}$ & 400 & $\mathrm{Nm}$ \\
Rated velocity & $n$ & 6000 & $\mathrm{rpm}$ \\
Slide weight & $m$ & 45 & $\mathrm{~kg}$ \\
Outer diameter of rotor & $r$ & 161.9 & $\mathrm{~mm}$ \\
Minimum coulomb friction torque & $T_{c}$ & 1.6 & $\mathrm{Nm}$ \\
Static friction torque & $T_{s}$ & 3.2 & $\mathrm{Nm}$ \\
Viscous friction torque & $T_{v}$ & 1.6 & $\mathrm{Nm}$ \\
Additional parameter & $\delta$ & 2 & - \\
Swing of the ripple torque & $F$ & 1.6 & $\mathrm{Nm}$ \\
Angular velocity of the ripple torque & $\omega_{0}$ & 900 & $\mathrm{rad} / \mathrm{s}$ \\
\hline
\end{tabular}

The desired trajectory in the simulations was set as follows:

$z^{*}(k+1)=\left\{\begin{array}{cc}100, & 0 \leq k<100 \\ 200, & 100 \leq k<300 \\ 150, & k \geq 300\end{array}\right.$

Selecting $a_{m}=1.321, b_{m}=-0.497, c_{m}=0.098$, and $d_{m}=0.078$, the reference model is described as follows:

$$
\begin{aligned}
\omega_{m}(k+1) & =1.321 \omega_{m}(k)-0.497 \omega_{m}(k-1) \\
& +0.098 \omega^{*}(k)+0.078 \omega^{*}(k-1)
\end{aligned}
$$

4.2 Control parameters selection and simulation analysis In the proposed control approach, the introduction of the positive weight factor $\lambda$ restricts the variations of control inputs and overcomes the steady state error. Theoretical analysis shows that the suitable option can guarantee the stability or improve the performance of the control system. Moreover, the orders $L_{u}$ and $L_{y}$ determine the usage of the region and degree of previous $\mathrm{I} / \mathrm{O}$ information. The simulation is started and performed under the following conditions, considering the two influence factors:

\subsubsection{Influence of weight factor $\lambda$}

For simplification, the first order of control law was employed and the gain coefficients were selected as $\alpha_{1}=\alpha_{2}=0.02$ and $\beta=0.004$. The simulation was started and performed with the following three conditions: $\lambda=0.1$, $\lambda=0.5$, and $\lambda=1$. Figure 2 shows the results and Table 2 lists the parameters.

Table 2. Simulation parameters

\begin{tabular}{c|c|c}
\hline$\lambda$ & System initial values & Controller parameters \\
\hline$\lambda=0.1$ & $y(1)=-1, y(2)=1$, & $\alpha_{1}=\alpha_{2}=0.02, \beta=0.004$, \\
or & $u(1)=0, \Delta u(1)=0$, & $L_{u}=L_{y}=1$, \\
$\lambda=2$ & $v(1)=v(2)=1, \theta(1)=2$ & $a_{i}=b_{j}=(1,0, \mathrm{~L}, 0)^{T}$ \\
or $\lambda=1$ & & \\
\hline
\end{tabular}
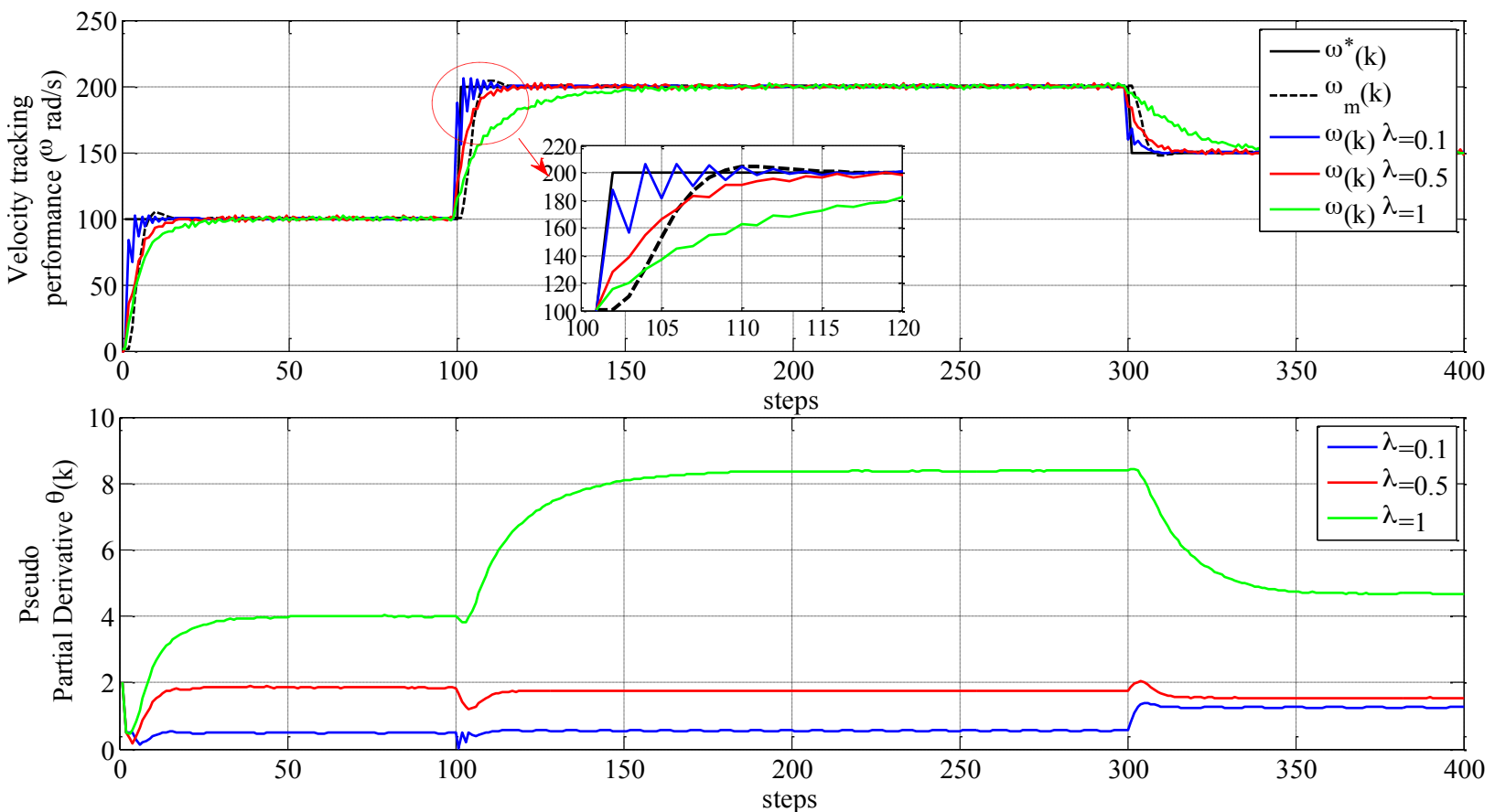

Fig. 2. Velocity responses with different values of $\lambda$

The values of weight factor $\lambda$ strongly influenced the system dynamic properties. The velocity overshoot decreased with increasing $\lambda$, indicating an improved disturbance rejection and reduced rapidity. When the value of weight factor $\lambda$ was less than 0.1 or greater than 10 , then the system presented poor performance. Therefore, stability and rapidity must be balanced when selecting the value of $\lambda$ in practical applications. Moreover, Figure 2 illustrates that PPD is a slow time-varying bounded parameter related to the system action point or system dynamics.

\subsubsection{Influence of the pseudo-orders $L_{u}$ and $L_{y}$}

The pseudo-orders $L_{u}$ and $L_{y}$ indicate the problem of how and/or how much to use the $\mathrm{I} / \mathrm{O}$ information. Exploiting a large amount of historical $\mathrm{I} / \mathrm{O}$ information can improve design accuracy. However, the excessive information will 
lead to high burden of the controlled system and low sensibility to the tuning of desired trail. The simulation was started with $\alpha_{1}=\alpha_{2}=0.02, \beta=0.004$, and $\lambda=1$, and performed with different values of pseudo-orders under the condition of nearest sample data of more than $40 \%$. Figure 3 shows that the robustness of the adaptive control is enhanced by exploiting additional historical information. Table 3 lists the parameters.

Table 3. Simulation parameters

\begin{tabular}{c|c|c}
\hline Orders & System initial values & Controller parameters \\
\hline \multirow{4}{*}{$L_{u}=L_{y}=1$} & $y(1)=-1, y(2)=1$, & $\alpha_{1}=\alpha_{2}=0.02, \beta=0.004$, \\
& $u(1)=0, \Delta u(1)=0$, & $\lambda=1$, \\
& $v(1)=v(2)=1, \theta(1)=2$ & $a_{i}=b_{j}=(1,0, \mathrm{~L}, 0)^{T}$ \\
\hline \multirow{5}{*}{$L_{u}=L_{y}=3$} & $y(1)=\mathrm{y}(2)=\mathrm{L}=\mathrm{y}(4)=1$, & $\alpha_{1}=\alpha_{2}=0.02, \beta=0.004$, \\
& $u(1)=\mathrm{u}(2)=u(3)=0$, & $\lambda=1$, \\
& $\Delta u(1)=\Delta u(2)=\Delta u(3)=0$, & $a_{i}=(0.6,0.2,0.2,0, \mathrm{~L}, 0)^{T}$ \\
& $v(1)=v(2)=v(3)=v(4)=1$, & $b_{j}=(0.6,0.2,0.2,0, \mathrm{~L}, 0)^{T}$ \\
\hline \multirow{5}{*}{$L_{u}=L_{y}=5$} & $\theta(1)=\theta(2)=\theta(3)=2$ & \\
& $y(1)=\mathrm{y}(2)=\mathrm{L}=\mathrm{y}(6)=1$, & $\alpha_{1}=\alpha_{2}=0.02, \beta=0.004$, \\
& $u(1)=u(2)=\mathrm{L}=u(5)=0$, & $\lambda=1$, \\
& $\Delta u(1)=\Delta u(2)=\mathrm{L}=\Delta u(5)=0$ & $a_{i}=(0.4,0.3,0.1,0.1,0.1,0, \mathrm{~L}, 0)^{T}$ \\
& $v(1)=v(2)=\mathrm{L}=v(6)=1$, & $b_{j}=(0.4,0.3,0.1,0.1,0.1,0, \mathrm{~L}, 0)^{T}$ \\
\hline
\end{tabular}

The simulation results in Figure 3 show that the system response is highly precise, has a small overshoot, and is highly stable when the introduction of additional historical $\mathrm{I} / \mathrm{O}$ information increases the amount of orders. However, using excessive historical information generates oscillations at the mutation instant of the desired output.

\subsection{Performance analysis in the case of data loss}

In the process of data sampling or data transmission, the control system inevitably emerges with problems of data loss or data incompletion. To analyze the output performance in this case, a variable was defined as follows:

$$
\begin{aligned}
& \gamma(k)=\left\{\begin{array}{ll}
1, & y(\mathrm{k}) \text { is not loss } \\
0, & y(\mathrm{k}) \text { is loss }
\end{array},\right. \\
& \bar{y}(k)=\gamma(k) y(k),
\end{aligned}
$$

where $\bar{y}(k)$ denotes the output in the data loss condition.

For simplification, we selected a first order system with data loss at the mutation instant and constant interval of the desired output, that is, $\gamma(k)=0$ when $95 \leq k \leq 105$ and $150 \leq k \leq 200$. Figure 4 illustrates the robustness of the proposed approach.
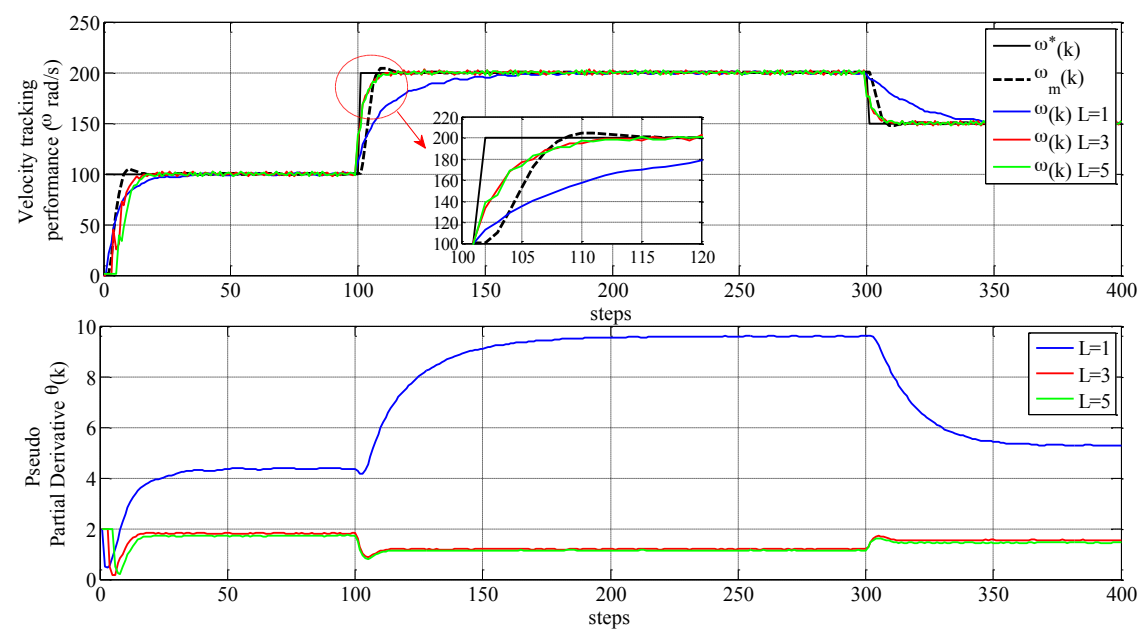

Fig. 3. Velocity responses with different $L_{u}$ and $L_{y}$ values
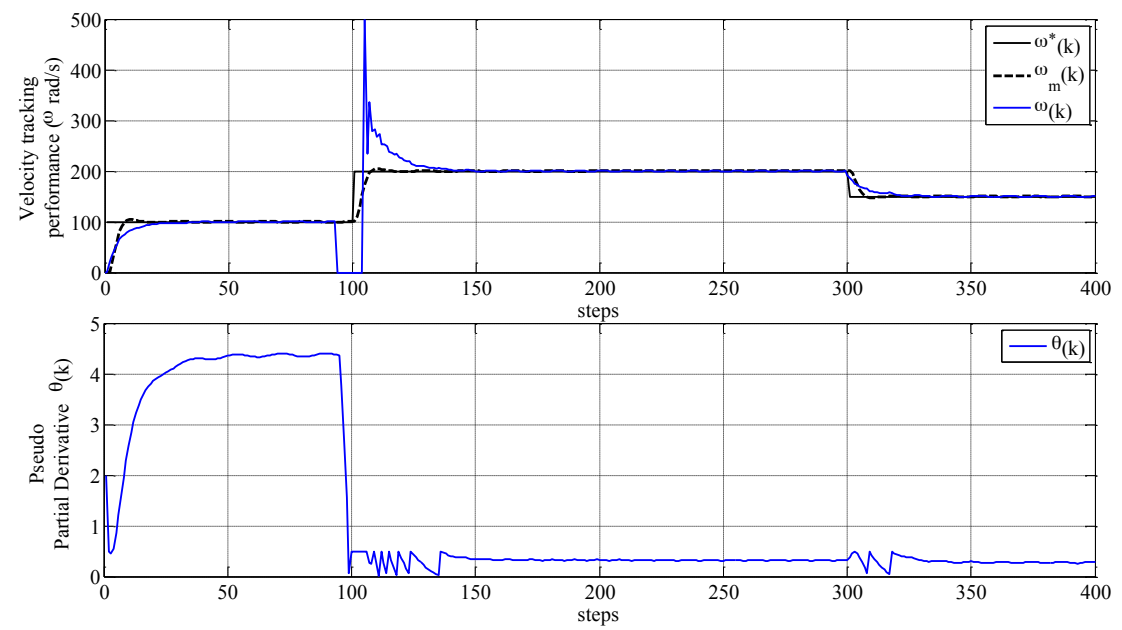

Fig. 4. Velocity responses in the case of data loss 
Simulation results in Figure 4 imply that the performance of the system emerged high oscillation and overshoot in the case of data loss at the mutation instant of the desired trail, and stability of the system were still guaranteed after normal data were recovered. On the contrary, the constant interval was partially influenced. To weaken the negative impact of data loss, some approaches, such as weighted average, adaptive filtering, and data prediction, can be involved to satisfy the accuracy demand.

The simulations indicated the following:

(1) The nonlinear PMSM system demonstrates satisfactory adaptability and stability under the proposed data-driven MRAC approach.

(2) The stability and rapidity of the PMSM system can be balanced by selecting the appropriate weight factors and orders. High values of $\lambda, L_{u}$, and $L_{y}$ indicate high stability for the controlled plant, whereas low values indicate favorable rapidity.

(3) Data loss or data incompletion causes large oscillation and overshoot at the mutation instant of the desired trail. High loss rates imply poor stability, but the data-driven MRAC approach ensures the convergence of the output error when normal data are recovered.

\section{Conclusions}

To improve the performance of traditional MRAC and suppress the influences of the modeling process, external disturbances, and unmodeled dynamics, an enhanced MRAC approach based on data-driven technique for PMSM was proposed. The equivalent linear model of PMSM was introduced in detail and the data-driven adaptive control law and the parameter estimate algorithm were presented. The following conclusions were obtained via theoretical analysis and simulation:

(1) The equivalent differential expression linear model, which was derived from I/O information, provides a highly general approach for the modeling process of nonlinear systems with uncertainties, thereby solving the problem of depending on the parametric model or structural information of the traditional MRAC approach.

(2) Favorable asymptotic convergence and improved disturbance rejection could be achieved through appropriate parameter coordination. The weight factor strongly influences the dynamic properties. High weight enhances the disturbance rejection and negatively affects the rapidity of the controlled system. Moreover, increasing pseudo-orders strengthens the robustness and fault tolerance. Furthermore, the high percentage of the nearest sample data means improved sensibility. Stability, rapidity, and disturbance rejection must be balanced when implementing the proposed approach according to different control targets.

(3) Data-driven MRAC, without support of the control plant model, designs the controller only with the I/O information and guarantees stability even if data are lost (not completely loss) or incomplete. When the loss rate is high, the oscillation and overshoot are high. Therefore, the integrity of the $\mathrm{I} / \mathrm{O}$ information determines the tracking accuracy of the approach, thereby putting forward high requirements for data detection and extraction.

The proposed data-driven MRAC approach solves the problem of depending on the parametric model or structural information of the PMSM drives and is especially useful for nonlinear motor systems with uncertainties. This approach provides a valuable reference for actual nonlinear systems that are typically difficult to model and control.

In spite of the progress to enhance the MRAC approach, the theoretical guidance or rules on the selection of parameter initial values and the general parameter presetting method remain the open issues to be addressed in the future.

This is an Open Access article distributed under the terms of the Creative Commons Attribution Licence

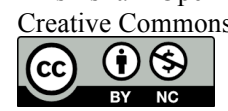

\section{References}

1. Tang R. Y., "Modern Permanent Magnet Machines-Theory and design". Beijing: China Mechanical Industry Press, China, 2016, pp. 1-4 2. Whitaker H.P., Yamron J., Kezer A., "Design of a model-reference adaptive control system for aircraft". Cambridge: Technical report, R164, MIT, Instrumentation Lab, USA, 1958, pp. 8.

3. Ortega R, Panteley E, "Comments on-adaptive control: stabilisation mechanism, existing conditions for stability and performance limitations". International Journal of Control, 87(3), 2014, pp. 581-588. 4. Landau I D, Lozano R, M'Saad M, Karimi A, "Adaptive Control: Algorithms, Analysis and Applications-2nd edition". Berlin: Springer Science \& Business Media, Germany, 2011, pp. 1-16.

5. Barkana, Itzhak, "Simple adaptive control-a stable direct model reference adaptive control methodology-brief survey". International Journal of Adaptive Control and Signal Processing, 28(7-8), 2014, pp. 567-603.

6. Landau I.D., Alma M., Martinez J.J., et al., "Adaptive Suppression of Multiple Time-Varying Unknown Vibrations Using an Inertial Actuator". IEEE Transactions on Control Systems Technology, 19(6), 2011, pp. 1327-1338.

7. Baldi, Ioannou, Mosca, "Multiple Model Adaptive Mixing Control: The Discrete-Time Case". IEEE Transactions on Automatic Control, 57(4), 2012, pp. 1040-1045.

8. Stepanyan V, Krishnakumar K, "Indirect M-MRAC for systems with time varying parameters and bounded disturbances". In: 2012 IEEE International Symposium on Intelligent Control (ISIC 2012), Dubrovnik, Croatia:IEEE, 2012, pp. 1232-1237.
9. Montanaro, Umberto, and J. M. Olm, "Discrete-time integral MRAC with minimal controller synthesis and parameter projection". Journal of the Franklin Institute, 352(12), 2015, pp. 5415-5436.

10. Gao Qing Zheng, and X. J. Xie, "Robustness analysis of discretetime indirect model reference adaptive control with normalized adaptive laws". International Journal of Automation and Computing, 7(3), 2010, pp.381-388.

11. Anderson R T, Chowdhary G, Johnson E N. "Comparison of RBF and SHL Neural Network Based Adaptive Control". Journal of Intelligent \& Robotic Systems, 54(1-3), 2008, pp. 183-199.

12. Kamalasadan S, Swann G D, Yousefian R, "A Novel SystemCentric Intelligent Adaptive Control Architecture for Power System Stabilizer Based on Adaptive Neural Networks". IEEE Systems Journal, 8(4), 2014, pp. 1074-1085.

13. Pathak K B, Adhyaru D M, "Performance analysis of neural network based MRAC". In: International Conference on Electrical, Electronics, Signals, Communication and Optimization, Visakhapatnam, India:IEEE, 2015, pp. 1-5.

14. Anwar A Z, Wang D, Muhammad A, "Fuzzy-Based Hybrid Control Algorithm for the Stabilization of a Tri-Rotor UAV". Sensors, 16(5), 2016, pp. 652.

15. Škrjanc Igor, B. Sašo, and M. Drago, "Direct fuzzy model-reference adaptive control". International Journal of Intelligent Systems, 17(10), 2010, pp. 943-963.

16. Costa R R, Liu H., "Robustness of VS-MRAC with respect to unmodelled dynamics and external disturbances". International Journal of Adaptive Control \& Signal Processing, 6(1), 2010, pp. 19-33. 
17. Hou Z S, Jin S T, "Model Free Adaptive Control: Theory and applications". Boca Raton: CRC Press, USA, 2013, pp. 9-11.

18. Hou Z.S. and Xu J.X., "On data-driven control theory: The state of the art and perspective". ACTA Automatica Sinica, 35(6), 2009, pp. 650667.

19. Wang J, Ji C, Cao L L, Jin Q B, "Model free adaptive control and parameter tuning based on second order universal model". Journal of Central South University (Science and Technology), 43(5), 2012, pp. 1795-1802.

20. Jin S T, Hou Z S, Chi R H, "A novel higher-order model-free adaptive control for a class of discrete-time SISO nonlinear systems". Journal of Dynamic Systems, Measurement, and Control, 135(4), 2013, pp. 044503.

21. F Qiao, Z Liu, J Wang, "Enhanced velocity tracking control using higher-order model-free adaptive approach for permanent magnet synchronous motor" . Journal of Engineering Science and Technology Review, 9(4), 2016, pp. 111-119.
22. Lin Cheng-Kai, Yen-Shin Lai, and Hsing-Cheng Yu, "Improved model-free predictive current control for synchronous reluctance motor drives". IEEE Transactions on Industrial Electronics, 63(6), 2016, pp. 3942-3953.

23. N. Dong, and Z. Chen, "A novel ADP based model-free predictive control”. Nonlinear Dynamics, 69(1-2), 2011, pp. 89-97.

24. Li X D, Xiao T F, Zheng H X, "Brief paper-Adaptive discrete-time iterative learning control for non-linear multiple input multiple output systems with iteration-varying initial error and reference trajectory". IET control theory \& applications, 5(9), 2011, pp. 1131-1139.

25. Freeman $\mathrm{C} T$, Tan $\mathrm{Y}$, "Iterative learning control with mixed constraints for point-to-point tracking". IEEE Transactions on Control Systems Technology, 21(3), 2013, pp. 604-616.

26. A. H. Brian, D. Pierre, C. D. W. Carlos, "A survey of models, analysis tools and compensation methods for the control of machines with friction”. Automatica, 30(7), 1994, pp. 1083-1138. 